\section{Dialogue: A blissful future for lupus nephritis: harnessing repeat kidney biopsies to identify meaningful biomarkers of disease}

\author{
Fernanda Payan Schober, ${ }^{1}$ William F Pendergraft $\mathrm{III}^{1,2}$
}

To cite: Schober FP, Pendergraft WF. Dialogue: A blissful future for lupus nephritis: harnessing repeat kidney biopsies to identify meaningful biomarkers of disease. Lupus Science \& Medicine 2015;2:e000083. doi:10.1136/lupus-2015000083

Received 2 March 2015 Accepted 5 March 2015

\section{SLinked}

http://dx.doi.org/10.1136/ lupus-2014-000061

\section{CrossMark}

\section{${ }^{1}$ Division of Nephrology, Department of Medicine, University of North Carolina (UNC) Kidney Center, Chapel Hill, North Carolina, USA \\ ${ }^{2}$ The Broad Institute of Harvard and MIT, Cambridge, Massachusetts, USA}

Correspondence to Dr Will Pendergraft; will_pendergraft@med.unc. edu
Kidney involvement in patients with systemic lupus erythematosus (SLE) portends a worse prognosis; thus, early and accurate detection of lupus nephritis is key. Unfortunately, current clinical markers, including creatinine clearance, autoantibodies, serum complement levels, urine sediment and proteinuria, do not always reliably identify patients with kidney disease. Moreover, these biomarkers do not help distinguish between various histopathological classes of lupus nephritis, which are often used as benchmarks to guide treatment. An intensely hot area of lupus research currently revolves around biased and unbiased approaches to identify biomarkers that can more reliably predict clinical and histopathological lupus-associated kidney activity and, perhaps even more importantly, durable response to treatment.

Novel biomarkers of lupus nephritis currently under investigation include but are not limited to B Lymphocyte Stimulator (BLyS), A Proliferation-Inducing Ligand (APRIL), tumor necrosis factor (TNF)-like WEAK inducer of apoptosis (TWEAK), and Monocyte Chemoattractant Protein-1 (MCP-1). BLyS, also known as B cell Activating Factor belonging to the TNF Family (or BAFF), is a transmembrane protein that is cleaved by a furin protease into a biologically active soluble protein that is important for $\mathrm{B}$ cell activation and differentiation. Numerous studies have shown that serum BLyS levels are elevated in patients with SLE compared with controls, making it the target of the recently Food and Drug Administration (FDA)-approved lupus drug, belimumab. ${ }^{1}$ Currently, the Belimumab International Lupus Nephritis Study clinical trial is underway to determine the efficacy of belimumab in patients with lupus nephritis (http://www.clinicaltrials.gov, NCT01639339). Like BLyS, APRIL is a member of the TNF family, and is thought to have a regulatory role in B cell proliferation. APRIL levels have been shown to be elevated in patients with lupus compared with healthy controls, although one study has found that levels may be lower in patients with lupus nephritis compared with patients who have lupus without kidney involvement. $^{23}$

TWEAK, a soluble cytokine expressed primarily by infiltrating leucocytes, is upregulated locally on epithelial and mesenchymal cells in injured and diseased tissues. It binds to its receptor, Fn14, to mediate proinflammatory responses, vascular activation, angiogenesis, cell growth, cell death, fibrogenic responses and progenitor responses. ${ }^{4}$ Urinary TWEAK levels have been found to be elevated in patients with lupus nephritis compared with patients with lupus and no kidney disease, making this a promising biomarker. ${ }^{5}$ Furthermore, the Anti-Tweak in Lupus Nephritis Patients clinical trial examining the efficacy of an anti-TWEAK monoclonal antibody in lupus nephritis is also underway (http://www.clinicaltrials.gov, NCT01499355). MCP-1 is a monocyte chemoattractant protein that induces release of lysosomal enzymes and generation of superoxide anions from monocytes and macrophages. Studies have found that urinary levels of MCP-1 are elevated in patients with lupus nephritis and reportedly increase during active disease and decrease with treatment. $^{6} 7$

In this issue of Lupus Science and Medicine, Parodis and colleagues evaluate serum BLyS and APRIL levels in 64 patients with active lupus nephritis (52 proliferative, 12 membranous) in Sweden. Levels of these two biomarkers were measured and followed throughout treatment as potential predictors of treatment response. Baseline BLyS levels were higher in patients with lupus nephritis than in controls and they remained unchanged even after induction treatment. A 
baseline BLyS level $<1.5 \mathrm{ng} / \mathrm{mL}$ predicted treatment response with a positive predictive value of $92 \%$. APRIL levels were higher in patients with lupus nephritis, but unlike BLyS levels, APRIL levels decreased following treatment. $^{8}$

These findings would suggest that APRIL levels mirror kidney disease activity and that low serum BLyS levels at baseline may portend improved prognosis. Interestingly, this study did what very few other studies involving biomarkers in lupus nephritis have ever done by comparing biomarker levels to kidney histopathology to determine disease activity and response to treatment rather than simply using a clinical score. An additional study in Thailand used protocol biopsies 6 months after treatment initiation and found that high serum APRIL levels corresponded with higher histological activity. ${ }^{9}$ Clearly, these thought-provoking findings need validation in locales other than Sweden and Thailand as well as in controlled trial settings. This is incredibly important to do as we determine the legitimacy of cytokine serum levels as predictors of kidney involvement, disease activity and response to treatment.

While we await validated biomarkers to assess disease activity and treatment efficacy, we must rely on the current gold standard, which is the kidney biopsy. There is interest in the lupus community regarding the role of protocol biopsies in the treatment of lupus nephritis, with limited published studies focused on repeat biopsies in this population. The perceived risk of complications, including pain, bleeding and even death, from kidney biopsies is often cited as being a contraindication to performing this procedure in patients who appear to be in remission. In addition, there is a reluctance to perform a biopsy in patients who we suspect are doing well, or who are at least clinically stable, because of this perceived risk. However, little is known about the actual risk that mandated protocol biopsies impose on patients with lupus as there have been few clinical trials that employ repeat kidney biopsies to evaluate treatment outcomes. We must therefore turn to the general nephrology literature, which reports complication rates ranging from $0.4 \%$ to $12.2 \% .^{10} 11$

One of the few trials to report complication rates of repeat kidney biopsy was the Mycophenolate Mofetil Versus Azathioprine for Maintenance Therapy of Lupus Nephritis (MAINTAIN) randomised controlled trial. Repeat protocol kidney biopsies were used to evaluate treatment response and 2 out of 60 biopsies (6.67\%) were associated with complications that were limited and did not require intervention. ${ }^{12}$ The study by Parodis and colleagues in this issue had an even lower complication rate of $3.1 \%$ with only four bleeding episodes out of 127 biopsies performed.

Repeat kidney biopsies are a useful tool to evaluate response to treatment and guide further intervention, particularly since there can be transformation in pathological classes, which could warrant changes in management. Changes in lupus class have been reported to occur in $25-75 \%$ of patients with lupus nephritis who undergo repeat kidney biopsies. ${ }^{13}{ }^{14}$ In one retrospective study, changes in morphological classes resulted in treatment regimen modifications in $87.3 \%$ of patients undergoing repeat kidney biopsies. ${ }^{15}$ Additionally, it has been demonstrated that clinical response does not always correlate with histological response. Patients who appear to be clinically quiescent can have active inflammatory lesions on repeat kidney biopsy. ${ }^{16}$ While proliferative changes would warrant a more aggressive treatment and increase in immunosuppression, repeat kidney biopsies can also detect significant fibrosis before kidney failure is detected, which could prompt practitioners to limit immunosuppression in patients whose kidney function is not likely to improve thereby decreasing complications associated with immunosuppression.

Great change is afoot in the field of lupus nephritis. As advances continue to be made in biomarker development for lupus nephritis through massive coordinated efforts to find biomarkers of durable treatment

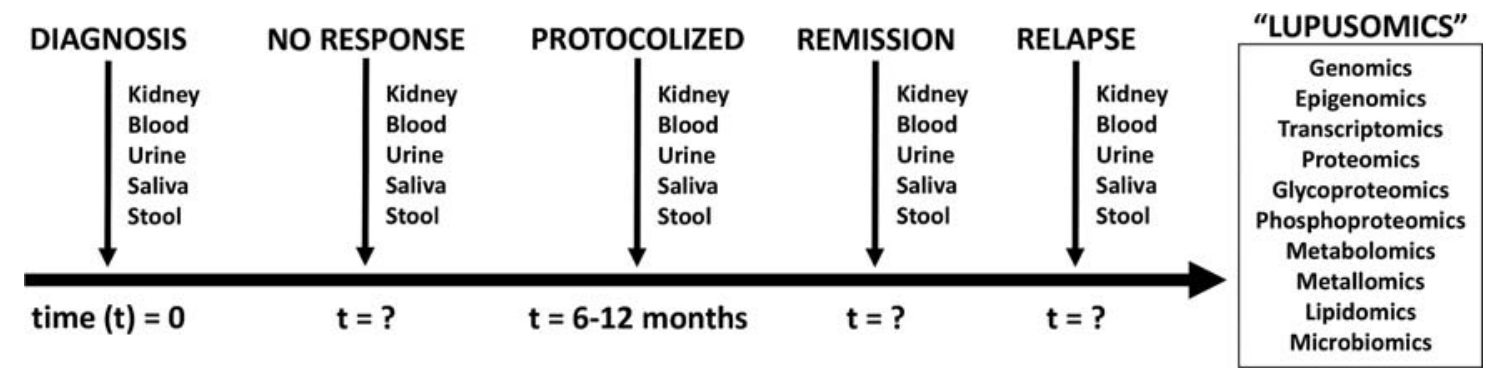

Figure 1 A proposed framework for harnessing repeat kidney biopsies in lupus nephritis to correlate clinical and histopathological disease states and identify meaningful biomarkers of disease. A kidney biopsy is performed at diagnosis and biosamples are obtained. If there is no response to remission induction therapy and/or relapse occurs after successful remission induction, a kidney biopsy could be performed and biosamples collected. If remission induction is achieved, repeat sampling could occur to assess histological quiescence, which could dictate next treatment steps and assist with prognosis regarding durable remission. In addition, a protocol biopsy could occur in all patients at a set time point to correlate clinical and histopathological findings, which could guide treatment duration. Biosamples could then be subjected to 'lupusomic' analyses as described at one or more time points to identify novel biomarkers of kidney involvement, disease activity and/or response to treatment. 
response, such as the National Institute of Health's newly formed Accelerating Medicines Partnership, repeat kidney biopsies at times of non-response, remission, relapse and even protocolised time points would validate potential candidates, and would assess for the presence of histological remission during times of presumed clinical quiescence (see figure 1). The potential fruit of the concept that one biomarker or a panel of biomarkers could replace the kidney biopsy would be an incredible step forward in the field. Until then, we should strongly consider adopting repeat kidney biopsies perhaps in a protocolised fashion rather than just when a flare is suspected. While this may be considered by some to be a more aggressive treatment approach, it could ultimately translate to improved outcomes in this vulnerable population.

Acknowledgements The authors thank the patients with lupus nephritis and all those dedicated to their care at UNC.

Contributors FPS and WFP3 conceived and outlined the manuscript collaboratively. FPS drafted the manuscript and WFP3 revised it critically for important intellectual content. FPS and WFP3 approved the final version to be published and agree to be accountable for all aspects of the work.

Funding FPS is supported by NIDDK grant T32 \#5T32DK007750-16. WFP3 is supported by grants from the NIDDK (P01-DK058335-14 and 1UM1-DK100845-01). WFP3 was also supported in part by NIDDK grant \# 5 F32 DK097891-02 and receives translational research funding from The Broad Institute.

Competing interests None.

Provenance and peer review Commissioned; internally peer reviewed.

Open Access This is an Open Access article distributed in accordance with the Creative Commons Attribution Non Commercial (CC BY-NC 4.0) license, which permits others to distribute, remix, adapt, build upon this work noncommercially, and license their derivative works on different terms, provided the original work is properly cited and the use is non-commercial. See: http:// creativecommons.org/licenses/by-nc/4.0/

\section{REFERENCES}

1. StohI W, Hilbert DM. The discovery and development of belimumab: the anti-Blys-lupus connection. Nat Biotechnol 2012;30:69-77.

2. Koyama T, Tsukamoto H, Miyani $\mathrm{Y}$, et al. Raised serum APRIL levels in patients with systemic lupus erythematosus. Ann Rheum Dis 2005;64:1065-7.

3. Vincent FB, Northcott M, Hoi A, et al. Association of serum B cell activating factor from the tumour necrosis factor family (BAFF) and a proliferation-inducing ligand (APRIL) with central nervous system and renal disease in systemic lupus erythematosus. Lupus 2013;22:873-84.

4. Michaelson JS, Wisniacki N, Burkly LC, et al. Role of TWEAK in Lupus Neprhitis: A bed-to-bedside review. J Autoimmun 2012;39:130-42.

5. Schwartz N, Rubinstein T, Burkly LC, et al. Urinary TWEAK as a biomarker of lupus nephritis: a multicenter cohort study. Art Res Ther 2009;1:R143.

6. Singh RG, Usha, Rathore SS, et al. Urinary MCP-1 as diagnostic and prognostic marker in patients with lupus flare. Lupus 2012;21:1214-18.

7. Rovin $\mathrm{BH}$, Song $\mathrm{H}$, Birmingham DJ, et al. Urine Chemokines as Biomarkers of Human Systemic Lupus Erythematosus activity. J Am Soc Nephrol 2005;16:467-73.

8. Parodis I, Zickert A, Sundelin B, et al. Evaluation of B lymphocyte stimulator and a proliferation inducing ligand as candidate biomarkers in lupus nephritis based on clinical and histopathological outcome following induction therapy. Lupus Sci Med 2015;2:e000061.

9. Treamtrakanpon W, Tantivitayakul P, Benjachat T, et al. APRIL, a proliferation-inducing ligand, as a potential marker of lupus nephritis. Arthritis Res Ther 2012;14:R252.

10. Hergesell O, Felten H, Andrassy K, et al. Safety of ultrasound-guided percutaneous renal biopy-retrospective analysis of 1090 consecutive cases. NDT 1998;13:975-7.

11. Preda A, Van Dijk LC, Van Oostaijen JA. Complication rate and diagnostic yield of 515 consecutive ultrasound-guided biopsies or renal allografts and native kidneys using a 14-gauge Biopsy gun. Eur Radiol 2003;13:527-30.

12. Houssiau FA, D'Cruz D, Sangle S, et al. Azathioprine versus mycophenolate mofetil for long-term immunosuppression in lupus nephritis: results from the Nephritis Trial. Ann Rheum Dis 2010;69:2083-9.

13. Singh A, Ghosh R, Kaur P, et al. Protocol biopsy in patients with lupus nephritis: A single center experience. Saudi J Kidney Dis Transpl 2014;25:801-7.

14. Lu J, Tam LS, Lai FM, et al. Repeat Renal biopsy in lupus nephritis: A change in histological pattern is common. Am J Nephrol 2011;34:220-5.

15. Greloni G, Scolnik M, Marin J, et al. Value of repeat biopsy in lupus nephritis flares. Lupus Sci Med 2014;1:e000004.

16. Zickert A, Sunderlin B, Svenungsson E, et al. Role of early repeated renal biopsies in lupus nephritis. Lupus Sci Med 2014;1:e000018. 